\title{
BMJ Open Functional outcome after Mason II-III radial head and neck fractures: study protocol for a systematic review in accordance with the PRISMA statement
}

\author{
Mårten Hagelberg, ${ }^{1}$ Alexandra Thune, ${ }^{1}$ Ferid Krupic, ${ }^{2}$ Björn Salomonsson, ${ }^{1}$ \\ Olof Sköldenberg ${ }^{1}$
}

To cite: Hagelberg M, Thune A, Krupic F, et al. Functional outcome after Mason II-III radial head and neck fractures: study protocol for a systematic review in accordance with the PRISMA statement. BMJ Open 2017;7: e013022. doi:10.1136/ bmjopen-2016-013022

- Prepublication history and additional material is available. To view please visit the journal (http://dx.doi.org/ 10.1136/bmjopen-2016013022).

Received 13 June 2016 Revised 17 November 2016 Accepted 1 December 2016



${ }^{1}$ Department of Clinical Sciences at Danderyd Hospital, Karolinska Institute, Stockholm, Sweden

${ }^{2}$ University of Gothenburg Institute of Clinical Sciences, Sahlgrenska Akademy, University of Gothenburg, Mölndal, Sweden

Correspondence to Dr Olof Sköldenberg; olof.skoldenberg@gmail.com

\section{ABSTRACT}

Introduction: Fractures of the radial head and neck are the most common fractures of the elbow, and account for approximately one-third of all elbow fractures. Depending on the fracture type the treatment is either conservative or surgical. There is no absolute consensus regarding optimal treatment for different fracture types. The aim of this protocol is to present the method that will be used to collect, describe and analyse the current evidence regarding the treatment of Mason II-III radial head and neck fractures.

Method and analysis: We will conduct a systematic review in accordance with the Preferred Reporting Items for Systematic Review and Meta-Analysis Protocol (PRISMA-P) guidelines statement. We will search a number of databases with a predefined search strategy to collect both randomised and nonrandomised studies. The articles will be summarised with descriptive statistics. If applicable a meta-analysis will be conducted.

Ethics and dissemination: Ethical approval is not required since this is a protocol for a systematic review and no primary data will be collected. The authors will publish findings from this review in a peer-reviewed scientific journal.

Trial registration number: CRD42016037627.

\section{BACKGROUND}

\section{Rationale}

Fractures of the radial head and neck are the most common fractures of the elbow, and account for approximately one-third of all elbow fractures. The estimated annual incidence of radial head and neck fractures are 2.8 per 10000 . The fractures often occur after indirect axial trauma following a fall onto an outstretched arm. The mean age of a patient who fractures their radial head or neck are between 44 and 48 and the male-to-female ratio is $2 / 3$. $^{1-4}$

\section{Strengths and limitations of this study}

- A review on this subject has never, to the best of our knowledge, been performed before according to Preferred Reporting Items for Systematic Review and Meta-Analysis (PRISMA) standard.

- Very common injury with clinical significance for patients.

- No clear consensus regarding optimal treatment.

- There are few randomised controlled trials on the subject.

- Heterogenic outcomes and methods across the literature possibly making comparisons difficult.

- Only studies in the English language will be included, thereby introducing a possibility of language bias.

The Mason classification is used to describe radial head and neck fractures. The classification is commonly divided into four groups and has been modified several times. According to the iteration by Broberg and Morrey, Mason I is a non-displaced fracture, Mason II is a fracture with more than $2 \mathrm{~mm}$ displacement, involving at least $30 \%$ of the radial head, Mason III fractures are significantly comminute and Mason IV is a fracture of the radial head or neck with associated elbow dislocation. Mason IV usually indicates greater trauma and greater soft tissue damage but is a very heterogenic group. It is a heterogenic group since both a minimally displaced and severely comminute fracture could be classified as Mason IV as long as the patient also has an elbow dislocation. There are no significant differences in age or gender disposition between the different Mason groups. ${ }^{3-6}$

The treatment of Mason I fractures is conservative with aspiration of the haematoma in the joint, a pressure bandage and sling for support, and active mobilisation as early as 
possible. There is currently no consensus on the treatment of patients with Mason type II fractures. Both conservative and surgical treatment is described with favourable outcome in the literature. Mason III-IV are treated in several ways, both open reduction internal fixation (ORIF) and arthroplasty are used as well as resection of the radial head. ${ }^{7-15}$

As described above, the treatment of radial head fractures is segmented. A few previous reviews have investigated the functional outcome after radial head fractures. However, the majority of these were conducted over 5 years ago and are only describing their results in descriptive ways.

To the best of our knowledge no standardised reviews according to the Preferred Reporting Items for Systematic Review and Meta-Analysis Protocol (PRISMA-P) have been published. ${ }^{16}$

The goal of this study is to summarise the outcome and treatment of radial head and neck fractures with a systematic review. The results are important for healthcare policymaking and patient care.

\section{Objectives}

This study will provide an overview of the recent published data on the subject of radial head and neck fractures classified as Mason II-III. A comparison of the functional outcome after different interventions including ORIF, arthroplasty, radial head resection and conservative treatment will be done. We aim to report the findings of this study in a way that makes it easy to use for clinical decision-making.

\section{METHODS AND ANALYSIS}

The proposed systematic review and this protocol will conform to the PRISMA-P guidelines and this protocol will be made publicly available before we initiate the review process. This study is also registered at the International Prospective Register of Systematic Reviews (PROSPERO) ${ }^{16}$

\section{Eligibility criteria}

Population

Studies with a population of 20 or more patients that includes patients with an age of 15 years or older with a traumatic Broberg-Morrey Mason II-III radial head or neck fractures are eligible for inclusion. There will be no upper limit on the follow-up time but reports with a mean follow-up time of $<1$ year are ineligible.

\section{Intervention}

Studies with patients that can be sorted into one or several of the following categories: conservatively treated patients, patients treated with ORIF, and arthroplasty or resection of the radial head are eligible for inclusion. If several treatments and/or Mason groups are represented in a study the patients will be subdivided and registered according to Mason classification and treatment received. Patients described to have associated injuries such as elbow dislocation or Essex-Lopresti injury will be excluded.

\section{Comparison}

Quantitative studies with a longitudinal design will be included, such as randomised controlled trials, cohort studies, cross-over studies, retrospective studies and casecontrol studies. Data will be collected regardless of the intervention received. Cross-sectional studies and case reports will be excluded. To minimise bias due to high drop-out, reports with a drop-out rate higher than $30 \%$ will not be taken into account. Only studies that use a Mason classification will be included. We will adapt the studies to the Broberg-Morrey iteration of the Mason classification.

\section{Outcome}

The primary outcome will be the participants' mean functional level measured with elbow and arm scores. Secondary outcomes will be complication rates, pain and range of motion.

\section{Search strategy}

The search strategy will be constructed by and in discussion with a librarian with expertise in healthcare databases and systematic reviews. We will search EMBASE, PubMed and the Cochrane library and limit the search to studies published in the English language during the past 30 years. The search strategy contains both Medical Subject Heading (MeSH) and non-MeSH terms. A less extensive presearch without review of the result will be carried out to calibrate the search strategy. Depending on the time consumption of the review process an update search to include all the latest articles might be conducted at the end of the review process. The search strategy for PubMed is included in online supplementary appendix 1 .

\section{Study records}

Search results are going to be saved and managed in Endnote V.X7 (Thomson Reuters, Philadelphia, Pennsylvania, USA). MH and AT will screen titles and abstracts of the found articles. Full text will be obtained of all articles that appear to meet, or if it is unclear if the article meets the predefined eligibility criteria. All exclusions and reasons for exclusion will be presented in a PRISMA flow chart together with the final review. ${ }^{16}$ All study data are going to be collected and managed using Research Electronic Data Capture (REDCap), an electronic data capture tool hosted at Karolinska Institute. ${ }^{17}$ REDCap is a secure, web-based application designed to support data capture for research studies, providing: (1) an intuitive interface for validated data entry; (2) audit trails for tracking data manipulation and export procedures; (3) automated export procedures for seamless data downloads to common statistical packages; and (4) procedures for importing data from external sources. 
The data to be extracted is presented in table 1 . Both reviewers will separately examine and extract data from the included studies, disagreement in the collected data will be resolved with discussion, if no consensus is reached a third reviewer (OS) will be consulted.

\section{Outcomes and prioritisation}

Several scores are anticipated to be used in the included studies. ${ }^{18}$ If a study reports the outcome in more than one score, we will prioritise as follows: Disabilities of the Arm, Shoulder and Hand (DASH), quick-DASH, Mayo Elbow Performance Score (MEPS) and Broberg and Morrey index. ${ }^{19} 20$ The scores will be modified to make comparison possible, for example, all scales will be modified so that a lower score equals a worse outcome. Complication rate includes non-union, wound infection, radial nerve injuries and reoperations. The complication rate will be measured as a percentage of patients included in the studies. We will also, if available, extract rated pain and range of motion.

\section{Risk of bias in individual studies}

Randomised controlled trials will be independently assessed by AT and $\mathrm{MH}$ regarding bias with the Cochrane Collaboration's risk of bias tool. This tool includes assessment of random sequence generation (selection bias), allocation concealment (selection bias), blinding of participants and personnel (performance bias), blinding of outcome assessment (detection bias), incomplete outcome data (attrition bias), selective reporting (reporting bias), baseline imbalance bias and other bias. ${ }^{21}$ To explore risk of bias in non-randomised studies the Newcastle-Ottawa scale will be used. The Newcastle-Ottawa scale has two different versions, one made to assess risk of bias in cohort studies and one made to assess case-control studies, the two versions differ slightly. The scale contains three categories: selection, comparability and exposure/outcome. These three categories are subdivided into $7-8$ items. ${ }^{22}$

\section{Data synthesis}

The collected data will be presented using appropriate descriptive statistics. If the available data permits, a meta-analysis will be conducted. We will subdivide and

\begin{tabular}{ll}
$\begin{array}{l}\text { Table } 1 \text { Data to be extracted } \\
\text { Publication } \\
\text { data }\end{array}$ & Publication year, author \\
\hline Study data & $\begin{array}{l}\text { Design, size of population, type of } \\
\text { intervention, mean duration of follow-up, } \\
\text { complication rate (including pain and } \\
\text { range of motion), drop-out rate, patient } \\
\text { reported and/or functional outcome score } \\
\text { (s), implant type } \\
\text { Mean age, female percentage, type of } \\
\text { fracture (classified according to Mason) }\end{array}$ \\
\hline
\end{tabular}

present the results according to the Mason group and intervention received. If a manageable amount of studies are found, we will also present the studies separately with all the extracted data. If this is not possible the data will be added as an appendix. A random-effects model will be applied as large heterogeneity regarding treatment conditions, participant characteristics and methodological factors are expected between included studies. A standardised mean difference with $95 \%$ CIs will be calculated to make comparison possible between studies that measure outcome with different rating scales. Dichotomous outcomes will be presented as risk ratios with 95\% CIs. If important data are missing, efforts will be made to contact the corresponding author. The analysis will be performed using R V.3.2.3 (R Foundation for Statistical Computing, Vienna, Austria), with the meta and metaphor packages. ${ }^{23}$

\section{Meta-biases}

We plan to assess the possibility of bias (publication bias, language bias and methodological biases) by plotting the included studies in a funnel plot. Funnel plot asymmetry will be examined using Eggers test of the intercept. $^{24}$

\section{Confidence in cumulative evidence}

The outcomes will be assessed regarding quality of evidence using the Grading of Recommendation Assessment, Development and Evaluation (GRADE). ${ }^{25}$ Consideration will be given to each of the GRADE criteria for assessing the quality of evidence. This approach grades the cumulative evidence to one of four categories: high, moderate, low or very low evidence. The GRADE approach takes eight items into account: study quality, inconsistency of result, indirectness of evidence, imprecision, publication bias, large magnitude of effect, effect of plausible residual confounding.

\section{DISCUSSION}

We have not found any systematic review examining this area with a published protocol according to PRISMA-P. Previously published systematic reviews suggest that there will be low evidence in the published data with few randomised controlled trails (RCTs). ${ }^{26-28}$ Owing to the lack of high-quality papers we will include both randomised and non-randomised studies. This approach enables a more comprehensive study of the available evidence regarding functional outcome after radial head and neck fractures.

As mentioned in the Methods and analysis section the Mason classification will be used in this review. This is a classification system with limitations since it has been revised several times. Some studies use the original three category classification while others use Broberg-Morreys or Hotchkiss four category iteration. The Hotchkiss and the Broberg-Morrey are quite similar and we will assume that a patient placed in a Hotchkiss group would be 
placed into the corresponding Broberg-Morrey group. This approach will in a few cases place the patients into wrong group introducing a limitation we will have to take into account when interpreting the results. A similar approach has previously been used by Kaas et $a l^{29}$

The intraobservability and interobservability when diagnosing radial head and neck fractures is not as good as one could wish for. This is a problem that several other fracture classification systems have as well such as the Neer classification of proximal humeral fractures. However, the Mason classification is the most commonly used in clinical and research settings and even though it has several shortcomings; it is currently the only practical way of studying radial head and neck fractures. ${ }^{30} 31$

When studying radial head and neck fractures, associated injuries such as elbow dislocation and Essex-Lopresti injuries are of great concern. We will exclude patients who are described to have associated injuries. Since a fracture of the radial head or neck with an elbow dislocation should be classified as Mason IV these patients will if correctly diagnosed not alter the results of this review. Essex-Lopresti is a complicating factor that is sometimes overlooked but it is quite uncommon and should be of minor impact of this review; Grassman et $a \hat{l}^{32}$ found 12 patients with Essex-Lopresti injury out of 295 patients with radial head fractures.

Stiffness, range of motion, pain and mechanical blockage are important measures of complication but not always reported in an adequate way. To be able to get information covering these factors we will as mentioned use DASH as our main outcome. DASH is a 30 -item questionnaire that includes three items covering pain and several questions covering stiffness and range of motion in an indirect manner. ${ }^{19} 29$

This is not the first review of this area but we believe that there is a need for an updated systematic review of this topic. A Cochrane study published 2013, only including RCTs, found three studies. With our review we will try to summarise more of the published studies available by also including other cohort studies. This will of course lower the possibility to draw firm conclusions but it will give a broader view of the available evidence. A study by Kaas $e t$ al was more thorough but is now 5 years old. We anticipate that by including recent publications we will be able to present the best available evidence regarding the best treatment of Mason II-III radial head and neck fractures. ${ }^{29} 33$

Acknowledgements The authors would like to express our sincere gratitude to librarian Alena Haarmann at the medical library of Danderyd hospital for constructing the search strategy.

Contributors $\mathrm{MH}$ is the main author of the protocol and will write the final report. MH and AT will be responsible for selection of articles and data extraction. OS supervised MH and AT, wrote the protocol and will write the final report. FK and BS was part of writing the revised protocol.

Funding The study was funded by the Karolinska Institute, Department of clinical sciences at Danderyd hospital.
Competing interests None declared.

Provenance and peer review Not commissioned; externally peer reviewed.

Open Access This is an Open Access article distributed in accordance with the Creative Commons Attribution Non Commercial (CC BY-NC 4.0) license, which permits others to distribute, remix, adapt, build upon this work noncommercially, and license their derivative works on different terms, provided the original work is properly cited and the use is non-commercial. See: http:// creativecommons.org/licenses/by-nc/4.0/

\section{REFERENCES}

1. van Riet RP, Morrey BF, O'Driscoll SW, et al. Associated injuries complicating radial head fractures: a demographic study. Clin Orthop Relat Res 2005;441:351-5.

2. Duckworth AD, Clement ND, Jenkins PJ, et al. Socioeconomic deprivation predicts outcome following radial head and neck fractures. Injury 2012;43:1102-6.

3. Kaas L, van Riet RP, Vroemen JPAM, et al. The epidemiology of radial head fractures. J Shoulder Elbow Surg 2010;19:520-3.

4. Mason ML. Some observations on fractures of the head of the radius with a review of one hundred cases. Br J Surg 1954;42:123-32.

5. Johnston GW. A follow-up of one hundred cases of fracture of the head of the radius with a review of the literature. Ulster Med $J$ 1962;31:51.

6. Broberg MA, Morrey BF. Results of treatment of fracture-dislocations of the elbow. Clin Orthop Relat Res 1987:109-19.

7. Esser RD, Davis S, Taavao T. Fractures of the radial head treated by internal fixation: late results in 26 cases. J Orthop Trauma 1995;9:318-23.

8. Geel CW, Palmer AK, Ruedi T, et al. Internal fixation of proximal radial head fractures. J Orthop Trauma 1990;4:270-4.

9. Khalfayan EE, Culp RW, Alexander AH. Mason type II radial head fractures: operative versus nonoperative treatment. J Orthop Trauma 1992;6:283-9

10. King GJW, Evans DC, Kellam JF. Open reduction and internal fixation of radial head fractures. J Orthop Trauma 1991;5:21-8.

11. Pearce MS, Gallannaugh SC. Mason type II radial head fractures fixed with Herbert bone screws. J $R$ Soc Med 1996:89:340P_4P.

12. Ring $D$. Open reduction and internal fixation of fractures of the radial head. Hand Clin 2004;20:415-27.

13. Duckworth AD, Wickramasinghe NR, Clement ND, et al. Long-term outcomes of isolated stable radial head fractures. J Bone Jt Surg Am 2014;96:1716-23.

14. Åkesson $\mathrm{T}$, Herbertsson $\mathrm{P}$, Josefsson $\mathrm{P}-\mathrm{O}$, et al. Primary nonoperative treatment of moderately displaced two-part fractures of the radial head. J Bone Jt Surg Am 2006;88:1909-14.

15. Antuña SA, Sánchez-Márquez JM, Barco R. Long-term results of radial head resection following isolated radial head fractures in patients younger than forty years old. J Bone Jt Surg 2010;92:558-66.

16. Shamseer L, Moher D, Clarke M, et al. Preferred reporting items for systematic review and meta-analysis protocols (PRISMA-P) 2015: elaboration and explanation. BMJ 2015;349:g7647.

17. Paul A Harris RT, Harris PA, et al. Research electronic data capture (REDCap) - a metadata-driven methodology and workflow process for providing translational research informatics support. J Biomed Inform 2008;42:377-81.

18. Longo UG, Franceschi F, Loppini $M$, et al. Rating systems for evaluation of the elbow. Br Med Bull 2008;87:131-61.

19. Hudak PL, Amadio PC, Bombardier C. Development of an upper extremity outcome measure: the DASH (disabilities of the arm, shoulder and hand) [corrected]. The Upper Extremity Collaborative Group (UECG). Am J Ind Med 1996;29:602-8.

20. Beaton DE, Wright JG, Katz JN, et al. Development of the QuickDASH: comparison of three item-reduction approaches. J Bone Jt Surg Am 2005;87:1038-46.

21. Higgins J, Green S. eds. Cochrane handbook for systematic reviews of interventions. Version 5.1.0 [updated March 2011]. The Cochrane Collaboration 2011. http://handbook.cochrane.org.

22. Wells G, Shea B, O'Connell D, et al. The Newcastle-Ottawa Scale (NOS) for assessing the quality of nonrandomized studies in meta-analysis. http://www.ohri.ca/programs/clinical_epidemiology/ oxford.asp (accessed 22 Apr 2016).

23. Viechtbauer $\mathrm{W}$, others. Conducting meta-analyses in $\mathrm{R}$ with the metafor package. J Stat Softw 2010;36:1-48.

24. Egger M, Davey Smith G, Schneider M, et al. Bias in meta-analysis detected by a simple, graphical test. BMJ 1997;315:629-34. 
25. Guyatt GH, Oxman AD, Vist GE, et al. GRADE: an emerging consensus on rating quality of evidence and strength of recommendations. BMJ 2008;336:924-6.

26. Miller G, Humadi A, Unni R, et al. Surgical management of Mason type III radial head fractures. Indian J Orthop 2013;47:323.

27. Li N, Chen S. Open reduction and internal-fixation versus radial head replacement in treatment of Mason type III radial head fractures. Eur J Orthop Surg Traumatol 2014;24:851-5.

28. Zwingmann J, Welzel M, Dovi-Akue D, et al. Clinical results after different operative treatment methods of radial head and neck fractures: a systematic review and meta-analysis of clinical outcome. Injury 2013;44:1540-50.
29. Kaas L, Struijs PAA, Ring D, et al. Treatment of mason type II radial head fractures without associated fractures or elbow dislocation: a systematic review. J Hand Surg 2012;37:1416-21.

30. Carofino BC, Leopold SS. Classifications in brief: the Neer classification for proximal humerus fractures. Clin Orthop Relat Res 2013;471:39-43.

31. Iannuzzi NP, Leopold SS. In brief: the mason classification of radial head fractures. Clin Orthop Relat Res 2012;470:1799-802.

32. Grassmann JP, Hakimi M, Gehrmann SV, et al. The treatment of the acute Essex-Lopresti injury. Bone Jt J 2014;96-B:1385-91.

33. Gao Y, Zhang W, Duan X, et al. Surgical interventions for treating radial head fractures in adults. Cochrane Database Syst Rev 2013: CD008987. 